\title{
A Multi-task Combinatorial Optimization Model Based on Genetic Algorithm and its Application in College Education Curriculum Planning
}

\author{
http://dx.doi.org/10.3991/ijet.v10i8.5218 \\ Jianying Li, Zhe Zhou and Liying Wang \\ Hebei Normal University of Science \& Technology, Qinhuangdao, Hebei, P.R. China
}

\begin{abstract}
Multi-task combinatorial optimization of a complex system is an important aspect of multi-task planning. To address the existing defects and limitations of the existing multi-task combinatorial optimization methods, the paper proposes a multi-task combinatorial model based on genetic algorithm. As a complex multi-task combinatorial optimization, the curriculum planning for higher education applies to itself the multi-task combinatorial model, which is based on genetic algorithm. Having fully considered such factors as teaching resources distribution, students' intention and teachers' intention, the paper designs a more efficient fitness function that has flexibly distributed courses and time in curriculum planning to meet the need of teaching in higher schools. Meanwhile, the paper utilizes specific cases of higher education to verify and analyze the algoritnm, and also carryies out a simulation test under the Matlab environment. the result indicates that the multi-task combinatorial optimization model based on genetic algorithm can relatively significantly optimize curriculum planning of higher education.
\end{abstract}

Index Terms-higher education, Curriculum planning, Genetic algorithm, Multi task, combinatorial optimization model.

\section{INTRODUCTION}

Combinatorial optimization is situated at the core of multi-task planning and is also nonlinear in a typical way. It involves many factors such as non-linearity, dispersion and uncertainty ${ }^{[1-3]}$. There are certain limitations for traditional mathematical tools in solving these problems, while genetic algorithm is highly capable of dealing with combinatorial optimization problems featured by high nonlinearity and dispersion ${ }^{[4-6]}$. Curriculum planning is one of the difficulties task in teaching affairs management ${ }^{[7-8]}$, since there are various restrictions requiring comprehensive consideration in aspects including classes, and classrooms, teachers, teaching hours, laboratories, and school buses cross school districts as well as holidays. Traditional manual curriculum planning entails large work load and may easily produce errors, and is especially timeconsuming for universities where there are too many class hours and courses to arrange. To solve this problem, researchers have currently applied several algorithms, including FP-growth algorithm for association rules, algorithm based on time-bitmap matching, algorithm based on resources matching, and group decision-making algorithm ${ }^{[9-11]}$. However, these algorithms usually fail to find the optimal solutions due to excess complexity of curriculum planning. Especially in recent years, as the enrollment of universities is expanding, the curriculum arrangement problems for higher education has become more serious. How to improve the curriculum arrangement efficiency and how to build a reasonable system for doing this have become researchable issues.

\section{CONCEPT OF GENETIC ALGORITHM}

Genetic algorithm (GA), first invented by American scholar J. Holland, is a random searching method that draws inspiration from biological evolution. By probability-based optimal searching, GA automatically obtains and guides the accumulation of the optimal search space and self-adaptively adjusts the direction of search. This algorithm possesses implicit parallelism and better global searching capacity. In recent years, GA is considered as an effective optimal searching tool, which has drawn more and more attention. Genetic algorithm targets at a group of feasible solution. The entire search space is traversed by selection, replication, cross-over and variation operations of the group of feasible solutions. Thus the population evolves constantly until the global optimal solution is obtained $^{[12-13]}$.

GA is a stochastic optimization and searching approach. Compared with traditional algorithms, GA has larger search space, faster convergence and lower requirement on initial data. It is a preferred tool for finding optimal solution.

\section{Multi-TASK COMBINATORIAL OPTIMIZATION MOdEl BASED ON GENETIC ALgORITHM}

\section{A. Initializing the population}

In multi-task planning, initialization is performed by stochastic search of idle space. For each task, an idle set idlehour (c) and a non-search space (nonsearchhour $(c)$ ) are reserved. For any $L_{c}(c \in C)$, let

$$
\text { nonsearchhour }(c)=\text { idlehour }(c)
$$

Then stochastic time $p \in$ nonsearchhour $(c)$ is generated. In case of conflict,

$$
\text { nonsearchhour }(c)=\text { nonsearchhour }(c)-1
$$

Stochastic search is continued in the idle space, otherwise

$$
\left\{\begin{array}{l}
\text { timelable }(c, p)=L_{c}(c \in C) \\
\operatorname{idlehour}(c)=\operatorname{idlehour}(c)-1
\end{array}\right.
$$


The above steps are repeated until the initial population of multi-task planning is generated.

\section{B. Building the fitness function}

During the evolution and search, GA essentially excludes external information, and is only based on fitness function. The search is based on the fitness value of each individual in the population. Thus, it is of vital importance in selecting fitness function, which directly determines the convergence speed of GA and the probability of finding the optimal solution using GA. The larger the value of fitness function is, the higher the probability of an individual being chosen. The fitness function for multi-task planning is designed as follows:

$$
F=\alpha_{1} \times T_{1}+\alpha_{2} \times T_{2}+\ldots .+\alpha_{n} \times T_{n}
$$

Where $T_{1}$ is the degree of satisfaction of task $1 ; T_{2}$ is the degree of satisfaction of task $2 ; T_{n}$ is the degree of satisfaction of task n; $\alpha_{1}, \alpha_{2}, \alpha_{n}$ are proportional coefficients, and $\alpha_{1}+\alpha_{2}+\ldots .+\alpha_{n=1}=1$.

\section{Selection, cross-over and variation models in multi- task planning}

Roulette wheel method is used in the present paper, that is, the probability of an individual being chosen is directly proportional to the fitness value. Suppose the size of the population is $\mathrm{M}$ and the fitness of an individual is $f_{i}$, then the probability of an individual being chosen is

$$
P_{i}=f_{i}, \sum_{i=1}^{M} f_{i}
$$

Obviously, the higher the fitness value is, the greater the probability of an individual being chosen, and vice versa. For those with higher fitness, the probability of replication and the number of replicated individuals are also higher. The expected number of replicated individuals is calculated by

$$
\overline{R_{i}}=f_{i} / \bar{f}_{i}
$$

To generate new, excellent individuals, multi-point cross-over is needed. Multiple points of cross-over are set randomly in individual string. Then mutual exchange takes place between cross-over points, thus producing two new offsprings at a time. A random number $\mathrm{r}(0<\mathrm{r}<1)$ is defined for the exchange operation at the exchange probability $P_{x}$. If

$$
r<P_{x}
$$

Then the exchange operation is implemented, otherwise, it is cancelled.

To ensure the genetic diversity of the population, which enables a larger search space, and to avoid the possibility of a too early evolution convergence or a local optimal solution on the chance that the genetic information is lost, the variation probability $P_{y}$ is defined. A random number $\mathrm{r}(0<\mathrm{r}<1)$ is generated for the variation operation. If

$$
r<P_{y}
$$

Then the exchange operation is implemented, otherwise, it is cancelled.

\section{Algorithm implementation}

The model and algorithm is implemented as follows:

Step 1: The tasks are encoded to generate the initial population which may have a very low fitness. It must be ensured that the gene value is within the specified interval. Set the terminative evolutionary algebra in genetic operation as $\mathrm{G}$, and the initial one $G_{0}=1$..

Step 2: The fitness function is determined. During the evolution and search, GA essentially excludes external information, and is only based on fitness function. The search is based on the fitness value of each individual in the population. Thus, it is of vital importance in selecting fitness function, which directly determines the convergence speed of GA and the probability of finding the optimal solution. The larger the value of fitness function is, the higher the probability of an individual being chosen.

Step 3: The fitness value of individuals is calculated according to the fitness function.

Step 4: Determine whether $G_{0}$ is larger than $G$. If so, then jump to step 8 , otherwise, move sequentially to step 5 .

Step 5: Determine whether the fitness values of the individuals meet the evolution requirements. If so, jump to step 7, otherwise, move sequentially to step 6 .

Step 6: Perform selection, cross-over and variation operations are performed to generate a new population. The evolutionary algebra is increased by 1 .

Step 7: Output the results.

Step 8: If the practical algebra is larger than the set one, then the evolution is considered as failed. The population is reinitialized, or the evolutionary algebra is increased.

\section{VerificAtion Through a CASE}

Curriculum arrangement for higher education can be seen as a complex system. There are various restrictions requiring comprehensive consideration in aspects including classes, classrooms, teachers, teaching hours, laboratories, school buses cross school districts as well as holidays. The paper thus utilizes specific cases of higher education to verify and interpret the aforementioned model and algorithm.

\section{E. Functional objectives of curriculum planning system}

The curriculum schedule is composed of five factors, namely, courses, class hours, grades, teachers and classrooms. An effective schedule should satisfy the 6 constraints listed as follows:

1. One teacher can be only teaching in one classroom at a time;

2. One teacher can be only teaching one course at a time;

3. Only one course is taught in one classroom at a time;

4. There must be only one teacher in one classroom at a time;

5. Only one course is taught to one class of students at a time;

6. There must be only one teacher giving lessons to one class of students at a time.

Several other non-rigid constraints need to be satisfied: 
1. The classes should be evenly arranged across the weeks. It should be avoided that some weeks are full of classes, while other weeks are run without classes.

2. The classes should be evenly arranged within the same week. The number of classes of the same course should be controlled within a day.

3. The teachers' wishes in the curriculum arrangement should be satisfied as far as possible

4. Commuting between different districts of campuses should be avoided to reduce the time spent on the road.

Curriculum of universities is divided into three types, namely, public compulsory course, specialized course and elective course. Students of different majors attend the public compulsory courses in the same lecture theater, and public compulsory courses usually have more class hours arranged. Therefore, the arrangement of public compulsory courses has to meet greater constraints. This type of courses should be arranged first. Specialized courses have fewer class hours and fewer students attending the class. Therefore, ordinary classrooms will do for these courses. This type of courses is arranged next. Elective courses have the least class hours, and the time and place of classes can be determined based on negotiation between teacher and students. This type of courses is arranged last. Here we mainly discuss the arrangement of specialized courses. Suppose two teachers are arranged for each specialized course, and the classes are given in the fixed classroom.

Based on the above constraints, the objectives of optimization are listed as follows:

1. The class hours should be evenly arranged on the whole.

2. The class hours of the same course should be arranged evenly within a week.

3. Teachers' opinions should be respected.

4. There must be zero conflicts, i.e.,

$$
f=\sum_{c \in c_{j}} v(c)=0
$$

where $C_{j}$ is the constraint set of the $\mathrm{j}^{\text {-th }}$ type; if $c$ constraint is satisfied, $v(c)=0$; if $c$ constraint is violated, $v(c)=1$.

\section{F. Encoding method}

Suppose that classes are given from Monday to Saturday, and the number of class hours is minimized for Saturday. One day is divided into 11 class hours, with 4 in the morning $(1,2,3,4), 4$ in the afternoon $(5,6,7,8)$ and 3 in the evening $(9,10,11)$. Each class hour is $50 \mathrm{~min}$, and two class hours combine into a larger class hour. Two class hours are separated by $5 \mathrm{~min}$ of rest, and two larger class hours are separated by $30 \mathrm{~min}$ of rest. The class time is $8: 00$ to $12: 00$ in the morning, $14: 00$ to $18: 00$ in the afternoon and 19:00 to 21:40 in the evening. Thus decimal encoding strategy is used. Each of the five factors of curriculum schedule is represented by a decimal number. For example, 051032118303 indicates the following information: major 051 , course No. 03, teacher No. 2, class No., classroom 303 of building A18, two teachers ar- ranged for this course. This encoding method is easy and covers every factor of curriculum.

\section{G. Initializing the population}

1. For initialization, all $6 \times 5$ larger class hours are selfstudy class.

2. The total class hours of each course are calculated, and the number and position of each chromosome (course) are determined. For example, 4 class hours are arranged for course 051032118303 in a week, and 2 chromosome 051032118303 are generated and located in the morning or in the afternoon; 3 class hours are arranged for course 163111103102, and 1 chromosome 163111103102 is generated and located in the evening.

3. The courses are arranged stochastically and without conflicts.

4. The above steps are repeated until 30 timetables are generated.

\section{H. Setting of fitness function}

The fitness function is constructed as follows:

$$
F=\alpha_{1} \times T_{1}+\alpha_{2} \times T_{2}
$$

Where $T_{1}$ is the degree of students' satisfaction with curriculum schedule (Table 1); $T_{2}$ is the degree of teachers' satisfaction with curriculum schedule (Table 2); $\alpha_{1}, \alpha_{2}$ are proportional coefficients and $\alpha_{1}+\alpha_{2}=1$. Here $\alpha_{1}$ is set as 0.6 , and $\alpha_{2} 0.4$.

TABLE I.

DEGREE OF STUDENTS' SATISFACTION WITH CURRICULUM SCHEDULE

\begin{tabular}{|l|c|}
\hline \multicolumn{1}{|c|}{ Arrangement of class hours for students } & Satisfaction degree \\
\hline $\begin{array}{l}\text { Two class hours of the same course in the same } \\
\text { day }\end{array}$ & 0 \\
\hline $\begin{array}{l}\text { Two class hours of the same course in every } \\
\text { other day }\end{array}$ & 2 \\
\hline $\begin{array}{l}\text { Two class hours of the same course in every } \\
\text { two days }\end{array}$ & 5 \\
\hline A total of 11 classes in one day & 0 \\
\hline A total of 9 classes in one day & 1 \\
\hline A total of 8 classes in one day & 2 \\
\hline Less than 8 classes in one day & 5 \\
\hline 4 classes on Saturday & 0 \\
\hline 2 classes on Saturday & 1 \\
\hline No classes on Saturday & 5 \\
\hline
\end{tabular}

TABLE II.

DEGREE OF TEACHERS' SATISFACTION WITH ARRANGEMENT OF TEACHING HOURS

\begin{tabular}{|l|c|}
\hline Arrangement of teaching hours for teachers & Satisfaction degree \\
\hline Most preferred arrangement & 5 \\
\hline Satisfied with the arrangement & 3 \\
\hline Do not like the arrangement & 1 \\
\hline Very unsatisfied with the arrangement & 0 \\
\hline
\end{tabular}

\section{Simulation results}

On the basis of the above selection, cross-over and variation models, and with the aid of GADS GA toolkit, the simulation experiment is carried out by adopting Matlab R2014a. The simulation parameters are: initial population 
30 , total number of generations 500 , exchange probability 0.5 , and variation probability 0.05 . The simulation results are shown in Fig. 1 and Fig. 2. Fig. 1 is the change of fitness over the course of 20 iterations. It can be seen that the fitness increases rapidly along with the increase of the iteration number; Fig. 2 is the GA model for curriculum arrangement. It can be seen that the algorithm converges to optimal solution after about 50 generations. The GA model is fast and highly efficient for solving the curriculum arrangement problem.

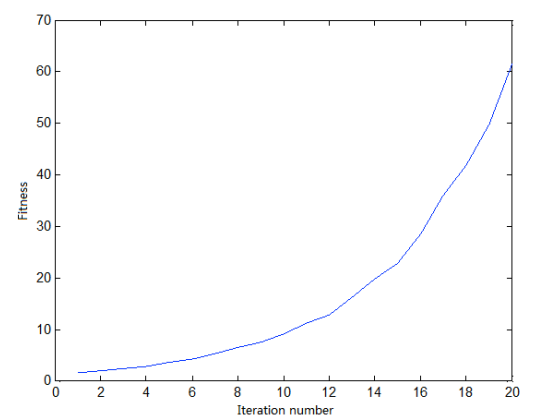

Figure 1. Changes of fitness during iterations for 20 times

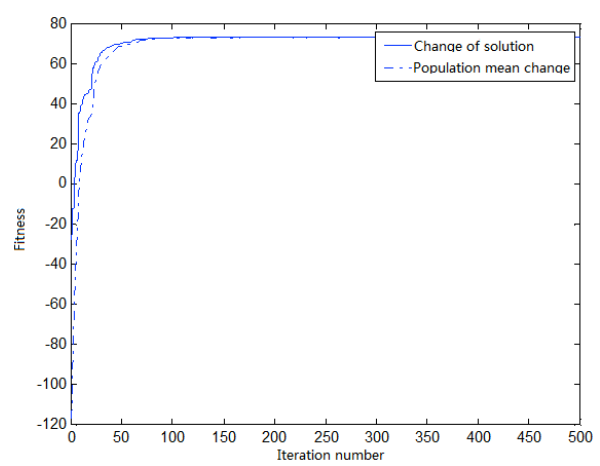

Figure 2. GA model for curriculum planning

\section{CONCLUSIONS}

To address problems in multi-task combinatorial optimization in complex systems, the paper proposes a multitask combinatorial optimization model based on GA. With this model, the paper researches on key contents such as the multi-task planning population initialization, the multitask planning construction of fitness function, and the model of selection, cross-over and variance in multi-task planning, and the implementation steps of multi-task combinatorial optimization model based on GA. Finally, the model and algorithm are verified and analyzed through a specific case of curriculum planning for higher education. The result indicates that this GA-based optimization model is effective and feasible for curriculum planning.

\section{REFERENCES}

[1] Chong WANG, Jun LI, Ning JING, Jun WANG, Hao CHEN. A Distributed Cooperative Dynamic Task Planning Algorithm for Multiple Satellites Based on Multi-agent Hybrid Learning [J] Chinese Journal of Aeronautics, 2011, 24(4): 493-505. http://dx.doi.org/10.1016/S1000-9361(11)60057-5

[2] Geiser Chalco Challco, Marco Aurélio Gerosa, Ig Ibert Bittencourt, Seiji Isotani. Automated instructional design for CSCL: A hierarchical task network planning approach [J]. Expert Systems with Applications, 2014, 41(8): 3777-3798. http://dx.doi.org/10.1016/ j.eswa.2013.12.016
[3] Spyros G. Tzafestas. 11 - Mobile Robot Path, Motion, and Task Planning [J]. Introduction to Mobile Robot Control, 2014: 429478.

[4] Stratis Kanarachos, Andreas Kanarachos. Intelligent road adaptive suspension system design using an experts' based hybrid genetic algorithm [J]. Expert Systems with Applications, 2015, 42(21): 8232-8242. http://dx.doi.org/10.1016/j.eswa.2015.06.047

[5] Oleg E. Bukharov, Dmitry P. Bogolyubov. Development of a decision support system based on neural networks and a genetic algorithm [J]. Expert Systems with Applications, 2015, 42(15-16): 6177-6183. http://dx.doi.org/10.1016/j.eswa.2015.03.018

[6] Dilay Cele. Inventory control in a centralized distribution network using genetic algorithms: A case study [J]. Computers \& Industrial Engineering, 2015, 87(12): 532-539.

[7] Ding Nianjin. Dominant values in curriculum planning [J]. Educational Research and Experiment, 2013, (2): 68-72.

[8] He Xuexin, Wu Huanhuan. Curriculum planning: motivation, dimension and path [J]. Journal of The Chinese Society of Education, 2011, (2): 36-40.

[9] Li Hongchan, Zhu Haodong. Decimal immunization GA used to solve UTP [J]. Systems Engineering-Theory \& Practice, 2012, 32(9): 2031-2036.

[10] Sun Min. Algorithm of arranging timetable based on time-bitmap matching $[\mathrm{J}]$. Journal of East China Shipbuilding Institute (Natural Science Edition), 2001, 16(6): 58-62.

[11] Sun Jianping, Yang Xiaoyong, Xiao Zhenghong. Usage of association rules in intellective system of arranging university curriculum schedule [J]. Computer Applications, 2002, 22(5): 34-37.

[12] Taehyoun Kim, Kwangkyu Lee, Jongmoon Baik. An effective approach to estimating the parameters of software reliability growth models using a real-valued genetic algorithm [J]. Journal of Systems and Software, 2015, 102(3): 133-144. http://dx.doi.org/10.1016/j.jss.2015.01.001

[13] Vivek Ahuja, Roy J. Hartfield, Andrew Shelton. Optimization of hypersonic aircraft using genetic algorithm [J]. Applied Mathematics and Computation, 2014, 242(9): 423-434. http://dx.doi.org/10.1016/j.amc.2014.05.120

\section{AUTHORS}

Jianying Li. She received her bachelor's degree of education in Hebei Normal University, Shijiazhuang, Hebei. (2005) and received master's degree of computer technology in Yanshan University, Qinhuangdao, Hebei. (2010). Now she is a lecturer in Hebei Normal University of Science \& Technology, Qinhuangdao, Hebei, P.R. China, 066004. Her current research interests include educational technology and vocational education. (email: 154157362@qq.com)

Zhe Zhou. She received her bachelor's degree of science in Langfang Teachers University, Langfang, Hebei. (2005) and master's degree of Educational Technology in Hebei University (2009). Now she is a lecturer in Hebei Normal University of Science \& Technology, Qinhuangdao, Hebei, P.R. China, 066004. Her major fields of study are instructional design, media teaching and distance education. (email: 30451344@qq.com)

Liying Wang. She received her bachelor's degree of education in Hebei Normal University, Shijiazhuang, Hebei. (2004) and master's degree of education in Tianjin normal university, Tianjin (2008). Now she is a lecturer in Hebei Normal University of Science \& Technology, Qinhuangdao, Hebei, P.R. China, 066004. Her current research interests include educational technology, data mining and vocational education. (40400149@qq.com)

This work was supported in Research topics of social development in Hebei province, the study which based on the regional economic development of Local colleges applied talents training. Issue number: 2015030401. Submitted 07 November 2015. Published as resubmitted by the authors 05 December 2015. 